\title{
OPTICAL PROPERTIES OF BLOWING SNOW
}

\author{
By J.W. POMERoY and D.H. Male
}

(Division of Hydrology, University of Saskatchewan, Saskatoon, Saskatchewan S7N OW0, Canada)

\begin{abstract}
Calculation procedures are developed and results shown for the exact calculation of extinction and meteorological visual range using the blowing-snow mass in the atmosphere and particle radius. Results of the calculations show: (1) For monochromatic radiation, geometrical optics approximations of the extinction efficiency are found to provide results of only moderate accuracy in calculating the extinction of radiation by a single particle. (2) For broad-band radiation, the geometrical optics approximation is sufficiently accurate for many single-particle measurement instruments and applications, except in the infra-red band where Mie theory should be used. (3) For typical blowing-snow particle-size distributions, the shape parameter of the distribution of particle radii and the mean particle radius are very important in broad-band extinction and visual-range modelling. Estimates of blowingsnow quantities from broad-band extinction measurements or visual range from blowing-snow quantities should address the shape and mean value of the snow-particle radius distribution.
\end{abstract}

\section{INTRODUCTION}

The optical properties of blowing snow can have great significance to mankind's endeavour in cold regions, as any person surviving travel during a "white-out" in a blizzard can attest. These optical properties also provide a means to estimate the physical properties of blowing snow. Considering the interest by scientists and engineers in the measurement of blowing snow using its attenuation of radiation (Landon-Smith and Woodberry, 1965; Schmidt, 1977, 1984; Gubler, 1981; Pomeroy and Male, 1985) and the prediction of visual range in blowing snow (Tabler, 1979, 1984), it is appropriate to model rigorously the optical characteristics of blowing snow.

Both the visual range for an object and the transmission of light can be defined in terms of the extinction of radiation over some path of transmission. Extinction of radiation occurs as blowing-snow particles scatter and absorb the radiant flux transmitted through an ensemble of these particles. Extinction is dependent on the wavelength of radiation $\lambda$, the radius of a sphere of equivalent area to the particle $r$, and the cumulative area of snow particles upon which the radiation is incident along its path, $N \pi r^{2}$, where $N$ is the number of particles. In visible light, the particle-size parameter $x=2 \pi r / \lambda$ is less than 50 for small blowing-snow particles. This indicates that blowing-snow particles in ambient light span the range of size from where the efficiency of extinction is variable to where it is relatively constant (van de Hulst, 1957). As extinction varies with the cumulative cross-sectional area of particles, it provides an indirect means to measure properties of blowing snow which control this area, such as the distribution of particle radii and drift density (mass of snow per unit volume of atmosphere).

Several investigations into the light-extinguishing characteristics of blowing snow have been published (Landon-Smith and Woodberry, 1965; Budd and others, 1966; Mellor, 1966; Schmidt, 1979; Tabler, 1979, 1984; Seagraves, 1981) which suggest or assume that extinction of light by blowing snow can be modelled using geometrical optics approximations. Geometrical optics assumes a uniform extinction efficiency for all particle sizes and for a beam of parallel radiation, and specifies that twice the radiant flux which intercepts the particle is actually extinguished. Errors may accrue from assuming this constant efficiency of extinction if small blowing-snow particles or wavelengths longer than the visible range are considered. Scattering is based on spherical particles in these approximations without addressing errors due to irregularity in particle shape. The applciations which attempt to relate the drift density (cumulative mass) to the cumulative cross-sectional area of the particles (Landon-Smith and Woodberry, 1965; Budd and others, 1966; Mellor, 1966; Seagraves, 1981) assume a uniform distribution of particle sizes and spherical particles. The work of Schmidt (1979) and Tabler (1979, 1984) relates the number of particles detected crossing a narrow beam of light to visual range, assuming spherical, non-absorbing particles of uniform size. Since distributions of blowing-snow particle sizes are of ten irregular and skewed (Budd, 1966; Schmidt, 1981), errors may accrue from assuming the mean particle mass $\left(r^{3} \rho_{\mathrm{i}}\right)$ or radius $(r)$ corresponds to the mean particle cross-sectional area $\left(r^{2}\right)$. Approaches which try to relate drift density or the number of particles to extinction should be cognizant of the magnitude of errors by:

(1) Irregular particle shape.

(2) Variable extinction efficiency.

(3) Irregular distribution of blowing-snow particle sizes.

In this paper, models are presented which describe the radiation-extinguishing characteristics of blowing-snow particles in the short-wave spectrum by applying Mie's electromagnetic scattering theory (Mie, 1908; as presented by van de Hulst (1957)) for the case where forward scattering of radiation is neglected. Mie theory calculations are more accurate but much more difficult than geometrical optics approximations. The differences in the results of the two calculation procedures are discussed for cases of single particles in both monochromatic and broad-band radiation, and for ensembles of particles, so that situations where the less time-consuming geometrical optics approximations are appropriate can be defined. The implications of Mie scattering are discussed in the context of selecting wavelengths for optical snow-particle counters and relating blowing-snow drift density, and the distribution of particle radii to the transmission of radiation and the meteorological visual range.

\section{PROPERTIES OF BLOWING SNOW}

Blowing snow is defined as surface snow which has been entrained and is being transported by the wind. The dominant mode of transport near and below a height of about $0.01 \mathrm{~m}$ is saltation, in which snow particles, accelerated by drag with the horizontal component of the wind, periodically rebound against the snow surface in a regular exchange of momentum. Above this height, suspended transport dominates, with particles supported by the vertical component of the wind and transported by the horizontal component. Suspended blowing-snow particles have appreciable terminal fall velocities, yet can be diffused by turbulence to heights of several tens of metres when wind speeds at $10 \mathrm{~m}$ exceed approximately $5 \mathrm{~m} / \mathrm{s}$ over exposed snow-covered terrain. Drift densities are at the maximum of 
approximately $1000 \mathrm{~g} / \mathrm{m}^{3}$ in the saltation layer, filling onethousandth of the atmospheric volume. Shiotani and Arai (1952), Budd (1966), and others have shown that there is an exponential decrease in the drift density as height is increased. Typically, drift densities at $2 \mathrm{~m}$ height are approximately $0.02 \mathrm{~g} / \mathrm{m}^{3}$, filling $0.2 \times 10^{7}$ th of the atmospheric volume.

The size distribution of blowing-snow particles is skewed towards smaller radii and varies with height and the degree of atmospheric turbulence. It has been fitted to the two-parameter gamma distribution for both the suspended (Budd, 1966) and saltating (Schmidt, 1981) modes of transport. This distribution has the form

$$
f(r)=\frac{r^{(\alpha-1)} \mathrm{e}^{(-r / \beta)}}{\beta^{\alpha} \Gamma(\alpha)}
$$

where $f(r)$ is the relative frequency of a particle of radius $r, \alpha$ is the distribution shape parameter, $\beta$ is the scale parameter, and $\Gamma$ denotes a gamma function. The particle radius is defined as the radius of a sphere of equivalent volume to that of the snow particle. The parameters $\alpha$ and $\beta$ are also defined in terms of the mean particle radius $\bar{r}$ (Haan, 1977) where

$$
\bar{r}=\alpha \beta .
$$

The value of $\alpha$ is approximately 5 for saltating snow. It increases to between 10 and 15 at a height of $2 \mathrm{~m}$. The mean particle radius decreases with height, typical values being $100 \mu \mathrm{m}$ near the surface and $40 \mu \mathrm{m}$ at $2 \mathrm{~m}$. The degree of turbulence in the wind can cause both $\alpha$ and $\bar{r}$ to vary at a given height.

\section{APPROXIMATING BLOWING SNOW AS ICE SPHERES}

Blowing-snow particles are usually metamorphosed fragments of the surface snow-pack. The particles are initially shattered from the snow-pack by the impact of already entrained particles and are then abraded by further impacts during saltation. Suspended particles are derived from saltating particles which are small enough to diffuse vertically. During suspended transport, the rate of sublimation is enhanced due to high particle ventilation rates (Schmidt, 1982). This process further rounds and smooths the particles. Figure 1 is a photograph of blowing-snow particles sampled at a height of $1 \mathrm{~m}$ above the snow-pack and shows the metamorphosed state of these fragments. A typical shape is slightly oblong and occasionally irregular with smooth edges (Schmidt (1981) has noted this as well) Schmidt (1984) and Gubler (1981) have commented that blowing-snow particles are much more amorphous than falling-snow particles, and thus are easily distinguished from the falling crystals. As blowing-snow particles are transported by often extreme turbulent fluctuations in th wind, it is unlikely that they are oriented; the observations of Schmidt (1984) with a double-beam particle counter confirm this. The density of blowing-snow particles has been assumed approximately equal to that of ice $\left(900 \mathrm{~kg} / \mathrm{m}^{3}\right)$ (Schmidt, 1981; Pomeroy and Male, 1987), because of thei metamorphosed state. Thus, blowing-snow particles approach the "optically ideal" ice sphere more closely than do other forms of snow.

The discussion in this paper of Mie theory and geometrical optics extinction of radiation by blowing snow is based on the premise that the particles behave optically as spheres of ice. Hodkinson (1963) extensively examined the influence of particle shape on scattering, as modelled in geometrical optics and Mie theory. His experiments with suspensions of variable-sized ground quartz particles show that for irregularly shaped particles greater than $1 \mu \mathrm{m}$ in visible light, measured extinction follows an average of Mie theory extinction. In terms of geometrical optics, Hodkinson concluded that diffraction by irregular, non-absorbing, randomly oriented particles is similar to that caused by spheres of equivalent cross-sectional area. External reflection is also similar as the probability of an angle of incidence is unchanged for randomly oriented particles. However, refraction as light leaves an irregular particle shows an angular deviation from that of a spherical particle. Pollack

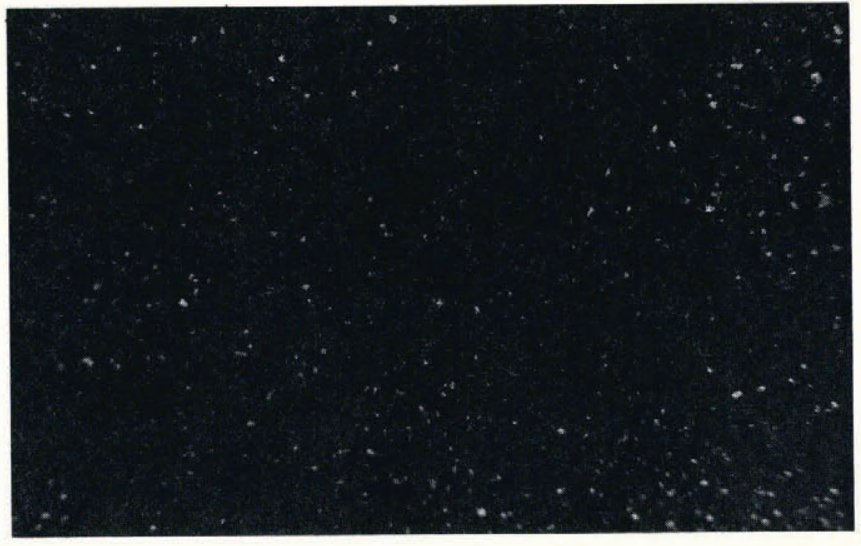

Fig. 1. Photograph of blowing-snow particles sampled at $1 \mathrm{~m}$ height. The width of the sample is $50 \mathrm{~mm}$. Atmospheric conditions: air temperature $-12^{\circ} \mathrm{C}$, relative humidity $62 \%$, wind speed at $1 \mathrm{~m}$ height $11 \mathrm{~m} / \mathrm{s}$; time and place $13.00 \mathrm{~h}$. 12 February 1985, Loreburn, Saskatchewan, Canada.

and Cuzzi (1980) provided a correction to scattering. of refracted and internally reflected radiation by non-spherical particles. This correction becomes negligible as the angle of scattering approaches zero.

Prominent atmospheric optical effects during the Canadian Prairie winter are "sun dogs" and halos around the Sun which can be observed when atmospheric ice (sharp crystal form) precipitates as crystals or as an ice fog, and "pillars" of light above street lamps which can be observed during snowfall when wind speeds are low and snow crystals are large. Over three winters of field observation in Saskatchewan, the authors have never observed sun dogs, halos, or pillars of light during blowing snow, only a diffuse appearance to the Sun or headlights of automobiles. This combination of optical phenomena is associated with light scattered by randomly oriented, non-uniform spheroids of size larger than the wavelengths of light.

Considering these observations and noting that blowingsnow particles are more spherical than Hodkinson's (1963) quartz crystals, it is suggested that the approximation of blowing-snow particles as spheres in the calculation of extinction results in small errors. This conclusion does not necessarily apply to cases where forward scattering must be accounted for.

\section{RADIATION EXTINCTION BY BLOWING SNOW}

Extinction of radiation by blowing snow may be considered for several cases: monochromatic radiation, individual particles; broad-band radiation, individual particles; and broad-band radiation, ensembles of particles. Results from examination of the first two cases are applicable to a blowing-snow particle detector which measures the drop in radiative transmittance associated with a single particle. The third case is applicable to measurement of large numbers of blowing-snow particles as in the transmissometer or optical attenuation meter. The third case can also be applied to problems of modelling visibility in blowing snow.

\section{Individual particles, monochromatic radiation}

The transmittance of radiation intercepted by a single particle is the ratio of the radiation transmitted in a forward direction after scattering and absorption by the particle to the radiation which would have been transmitted without interception by the particle. In this paper, all radiation scattered and absorbed is considered unavailable for transmission in a forward direction, i.e. no scattered radiation is detected. This is common where the angle between the particle centre and detector diameter is very small.

The extinction efficiency $Q_{\text {ext }}$ is the ratio of the radiant flux scattered and absorbed by a particle to that 
which actually intercepts the particle surface (dimensionless) The variation of the extinction efficiency with the particle radius and incident wavelength must be defined to determine the transmittance associated with a particular particle. In geometrical optics, $Q_{\text {ext }}$ is considered equal to 2.0. This implies a quantity of radiation equal to that intercepting the particle surface is extinguished via refraction, reflection, and absorption, and an equal amount by Fraunhoffer diffraction by the particle edge. This approximation is only valid for particles which are large with respect to the incident wavelength. For smaller particles, the extinction efficiency varies substantially and is a function of the particle-scattering parameter $x=2 \pi r / \lambda$ and the complex index of refraction. As some blowing-snow particle sizes lie within the range where the extinction efficiency becomes variable, it is of interest to examine the more precise Mie theory predictions of $Q_{\text {ext }}$. Mie (1908) employed electromagnetic theory to describe rigorously the perturbation of a planar coherent wave by spherical particles. van de Hulst (1957) detailed the Mie theory calculations of extinction efficiency for transparent, partially absorbing, and opaque spheres. The full Mie calculation is tedious and even the fastest algorithms (Wiscombe, 1980 Ungut and others, 1981) require extensive computing time, particularly when $x$ is large and a range of size parameters is considered. Nussenzveig and Wiscombe (1980) proposed asymptotic approximations to the extinction efficiency with calculations based on Mie theory. These calculations exhibit errors of less than $0.01 \%$ for large-size parameters when compared with exact Mie calculations and are well suited to applications where an ensemble of non-uniform size particles is considered.

Warren (1984) has compiled the real and imaginary components of the refractive index of ice, for various wavelengths. Approximating blowing-snow particles as ice spheres of equivalent cross-sectional area, the extinction efficiency has been calculated for various particle sizes and incident wavelengths using Nussenzveig and Wiscombe's (1980) algorithm. The results, for monochromatic radiation, are plotted against snow-particle radius for various wavelengths in Figure 2. The wave pattern in $Q_{\text {ext }}$ is a manifestation of interference phenomena amongst various orders of scattering rays. Figure 2 suggests that when extinction of monochromatic radiation by individual snow particles is considered (such as the case of a laser particle counter in which the detector receives no forward scattering), appreciable variation in the extinction efficiency for particles of similar size can occur. This effect is most pronounced for smaller particles and longer wavelengths, for example, a wave-pattern amplitude of greater than $15 \%$ is manifest by particles under $19 \mu \mathrm{m}$ radius extinguishing radiation with a wavelength of $2 \mu \mathrm{m}$.

To illustrate precisely where unacceptable differences exist between the geometrical optics extinction efficiency, $Q_{\text {ext }}=2.0$, and the Mie theory predictions, Table I indicates the smallest snow-particle radii for which differences of less than $1-5 \%$ occur for various monochromatic wavelengths. Particles smaller than the radii listed can result in differences exceeding the stated limit. When variations from the geometrical optics extinction efficiency of 2.0 exceed $5 \%$, the wave pattern in $Q_{\text {ext }}$ makes relating the extinction by an individual particle to its radius extremely difficult, even with Mie theory calculations. An irregularity in particle shape will offset the wave pattern in $Q_{\text {ext, }}$, compounding the difficulties. For differences less than $5 \%$, calibration of a snow-particle detector using Mie theory approximations is appropriate if the errors in using geometrical optics are tolerable. An example of the application of Table $I$ is: if the designer of a monochromatic optical particle detector has set an error tolerance of $3 \%$ in his theoretical calibration, and wishes to estimate the size of particles with radii as small as $20 \mu \mathrm{m}$, he can use the $300 \mathrm{~nm}$ with geometrical optics but not the $600 \mathrm{~nm}$ wavelength. If the designer is willing to calibrate the device with Mie theory approximations he can use the $600 \mathrm{~nm}$ wavelength but will exceed the $5 \%$ limit and enter essentially unpredictable ranges of $Q_{\text {ext }}$ for the 1.06 and $2 \mu \mathrm{m}$ wavelengths.

\section{Individual particles, broad-band radiation}

When radiation possessing a range of wavelengths is transmitted, the wave patterns shown in Figure 2 average
TABLE I. SMALLEST SNOW-PARTICLE RADII $(\mu \mathrm{m})$ FOR WHICH THE DIFFERENCE BETWEEN THE EXTINCTION EFFICIENCY CALCULATED USING APPROXIMATIONS TO THE MIE THEORY AND THAT CALCULATED USING GEOMETRICAL OPTICS IS LESS THAN 1, 2, 3 4, AND 5\%, RESPECTIVELY, FOR MONOCHROMATIC RADIATION

\% Difference

Wavelength

$\begin{array}{rrrrr} & 300 \mathrm{~nm} & 600 \mathrm{~nm} & 1.06 \mu \mathrm{m} & 2 \mu \mathrm{m} \\ 1 & 48 & 95 & 170 & >300 \\ 2 & 22 & 48 & 84 & 124 \\ 3 & 17 & 30 & 54 & 87 \\ 4 & 6 & 20 & 37 & 62 \\ 5 & 5 & 12 & 32 & 53\end{array}$

out somewhat. Hodkinson (1963) noted that the wave patterns also average out when radiation is scattered by an ensemble of irregularly shaped particles. Broad-band radiation possesses a range of wavelengths, and its extinction by blowing-snow particles will follow the mean of the wave patterns. To determine a mean Mie extinction efficiency, a particle radius and one of four central wavelengths are used to calculate the particle-size parameter $x=2 \pi r / \lambda$. The Mie approximation of extinction efficiency is then calculated using an appropriate refractive index for ten wavelengths within $\pm x / 6$ of each $x$ and the mean of these values taken as the mean Mie extinction efficiency for the particle radius. This variation in $x$ is equivalent to a $100 \mathrm{~nm}$ range of wavelength for a mean wavelength of $300 \mathrm{~nm}$, increasing to a $660 \mathrm{~nm}$ range at $2 \mu \mathrm{m}$. The Mie extinction efficiency for a single particle in broad-band radiation (mean Mie extinction efficiency) is shown in Figure 3 plotted against the particle radius, for four central wavelengths. Note that the smoothing of extinction efficiencies becomes less pronounced as the wavelength increases. It is clear that, even for radiation possessing a range of wavelengths, errors of a notable magnitude can occur when approximating the extinction efficiency of a single blowing-snow particle with the geometrical optics value. Thus, the total radiation extinction by a single particle is not necessarily uniquely associated with a particular radius.

Designers of optical particle counters should be aware of the variation of the extinction efficiency when selecting wavelength and band width of radiation, and in interpreting measurements by these devices. For broad-band radiation as calculated in this section, Table II shows the smallest particle radii for which the difference between the Mie theory and geometrical optics $Q_{\text {ext }}$ are less than $1-5 \%$ respectively for various central wavelengths. While comparison of broad-band values with Table $\mathrm{I}$ indicates considerable improvement over the monochromatic case, there are still ranges of particle size and wavelength where the errors of using geometrical optics may exceed the design limits of snow-particle detectors. A significant advantage of broad-band radiation is that for mean wavelengths less than $2 \mu \mathrm{m}$, the extinction efficiency can be predicted for a particle radius. For these wavelengths, selection of the calculation procedure for calibration and the central wavelength to be employed can be based entirely on the acceptable level of error for gauge calibration. For the example of the particle-detector design in the last section, a further option is to use broad-band radiation and the appropriate central wavelength as chosen using Table II.

\section{Ensemble of particles, broad-band radiation}

The electromagnetic transmittance through an ensemble of randomly located particles is the ratio of the radiant flux transmitted through this mixture to that flux transmitted without the scattering and absorption effects of the particles. This is the transmittance measured by an optical detector which receives no forward-scattered light. Following the Bouger-Lambert law, the transmittance $\tau$ through an ensemble of particles smaller than the beam of radiation is found from the length of transmission $l$ and the radiation 

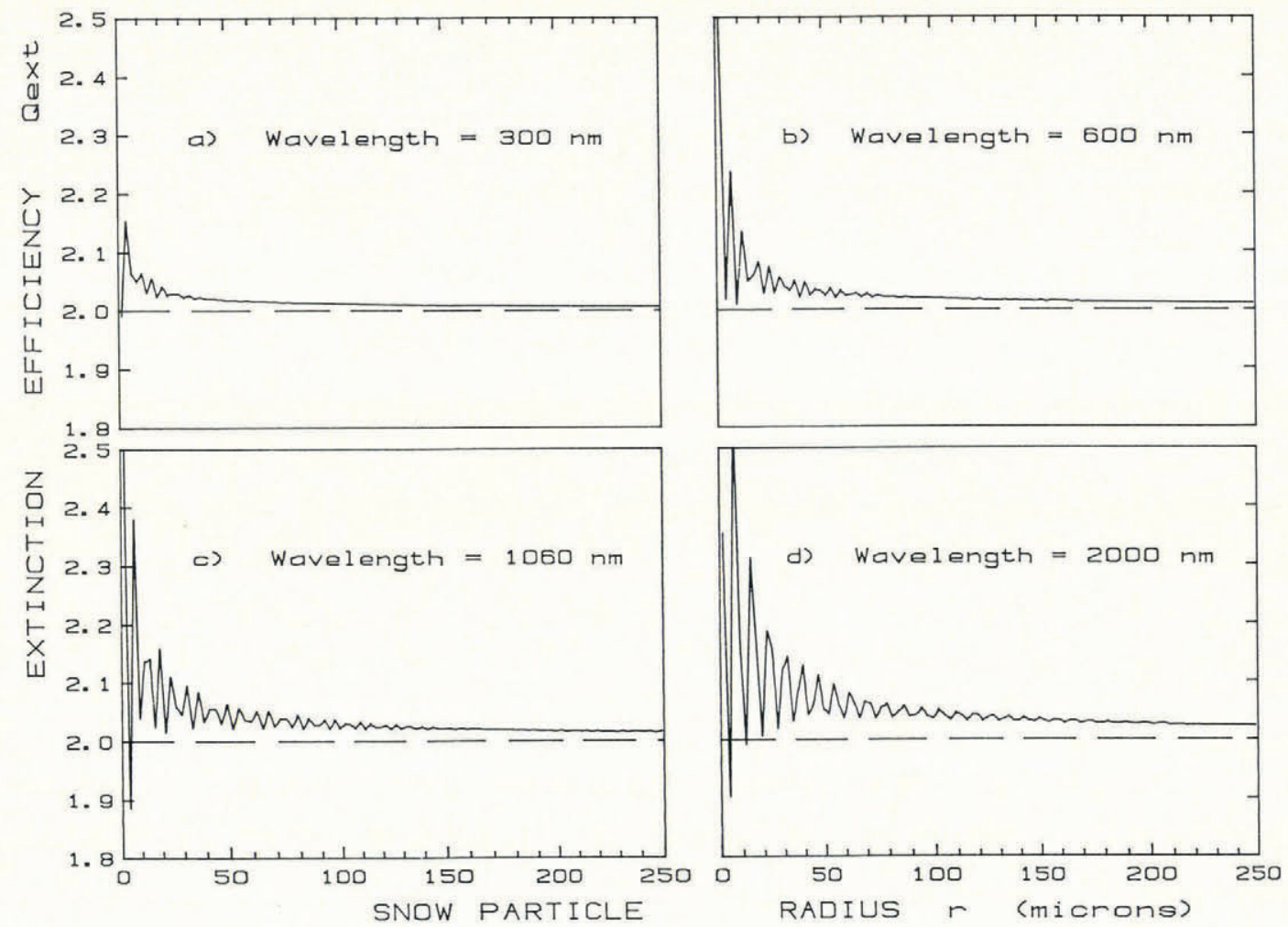

Fig. 2. Mie extinction efficiencies calculated for individual blowing-snow particles in monochromatic radiation at wavelengths of (a) $300 \mathrm{~nm}$, (b) $600 \mathrm{~nm}$, (c) $1.06 \mu \mathrm{m}$, (d) $2.0 \mu \mathrm{m}$. Note the geometrical optics approximation of 2.0. Wave forms are due to diffraction patterns.
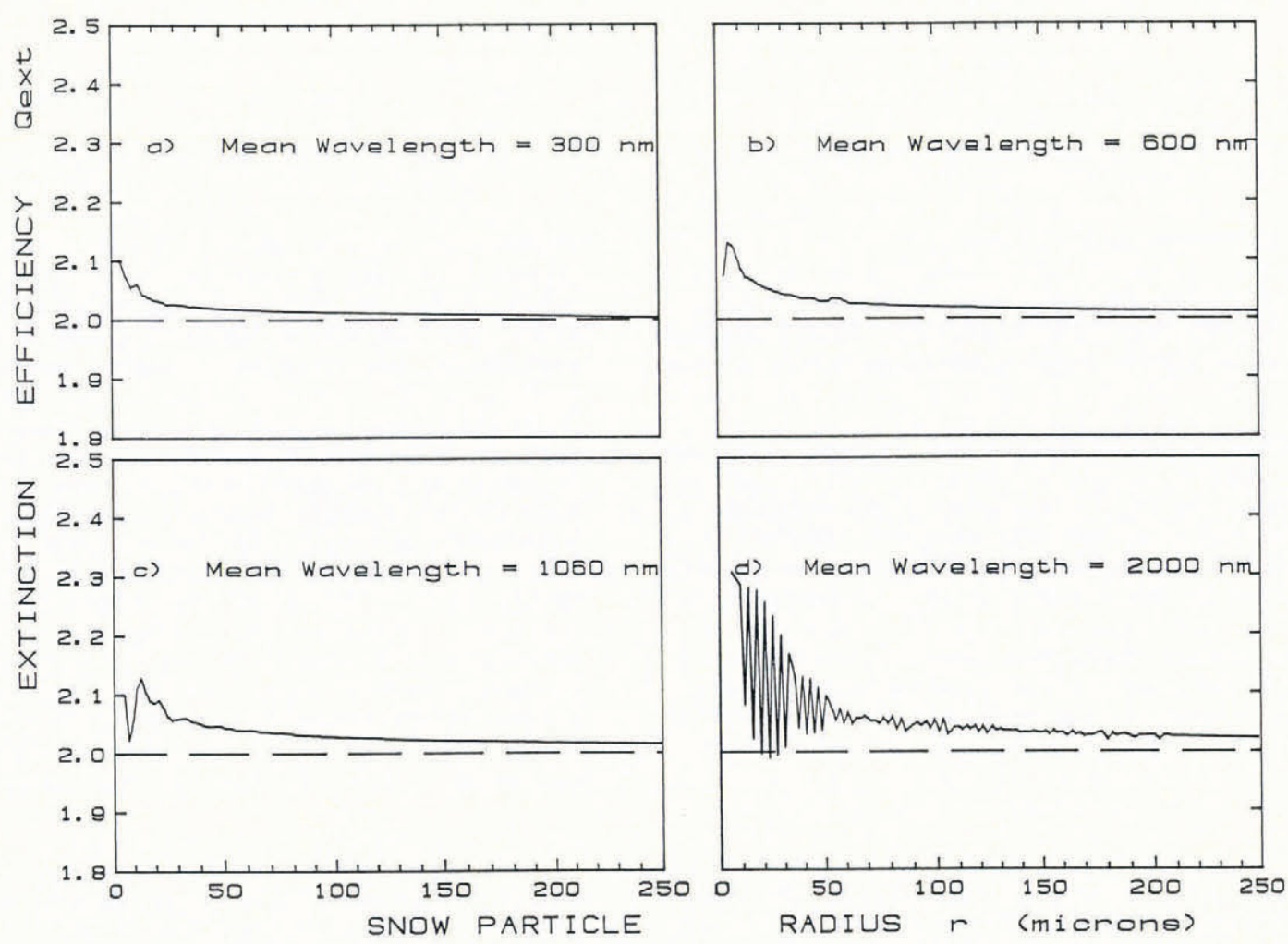

Fig. 3. Mie extinction efficiencies calculated for individual blowing-snow particles in broad-band (width $=x / 3$ ) radiation at wavelengths of (a) $300 \mathrm{~nm}$, (b) $600 \mathrm{~nm}$, (c) $1.06 \mu \mathrm{m}$, (d) $2.0 \mu \mathrm{m}$. Note the subdued wave forms and closer correspondence to the geometrical optics approximation of 2.0 compared with Figure 2. 
TABLE II. SMALLEST SNOW-PARTICLE RADII $(\mu \mathrm{m})$ FOR WHICH THE DIFFERENCE BETWEEN THE EXTINCTION EFFICIENCY CALCULATED USING APPROXIMATIONS TO MIE THEORY AND THAT CALCULATED USING GEOMETRICAL OPTICS IS LESS THAN 1, 2, 3, 4, AND 5\%, RESPECTIVELY, FOR BROAD-BAND RADIATION

\% Difference

$\begin{array}{lrrrr} & 300 \mathrm{~nm} & 600 \mathrm{~nm} & 1.06 \mu \mathrm{m} & 2 \mu \mathrm{m} \\ 1 & 47 & 94 & 166 & 314 \\ 2 & 16 & 31 & 62 & 136 \\ 3 & 13 & 18 & 34 & 81 \\ 4 & 5 & 11 & 24 & 55 \\ 5 & 4 & 9 & 16 & 50\end{array}$

extinction coefficient $\mu_{\lambda}$ for the wavelength $\lambda$ considered, where

$$
\tau=\mathrm{e}^{\left(-\mu_{\lambda} l\right)} .
$$

In this application, the small particles are particles of blowing snow, the mixture is composed of snow and air, and the length of transmission refers to distance of transmission through the atmosphere.

The extinction coefficient is the relative radiant flux which is scattered and absorbed per unit volume of the medium and has units of $l^{-1}$. All radiation scattered and absorbed is considered unavailable for transmission in its original direction. The extinction coefficient is a property of blowing snow and can be derived from the optical effects of individual snow particles if a quantity of blowing snow can be described in terms of these particles. Thus, for a non-uniform distribution of particle sizes,

$$
\mu_{\lambda}=\pi N \int f(r) r^{2} Q_{\text {ext }}(r, \lambda) \mathrm{d} r .
$$

In Equation (4), $N$ is the number of snow particles per unit volume of atmosphere, and $Q_{\text {ext }}(r, \lambda)$ denotes the particleextinction efficiency, a function of particle radius and incident wavelength.

The extinction coefficient can be expressed in terms of the drift density, the particle-size distribution, and extinction efficiency by the following substitution. The drift density $\rho_{\mathrm{d}}$ is defined as

$$
\rho_{\mathrm{d}}=4 / 3 \pi \rho_{\mathrm{p}} N \int f(r) r^{3} \mathrm{~d} r
$$

where $\rho_{\mathrm{p}}$ is the density of blowing-snow particles, considered equivalent to that of ice. Solving Equation (5) for $N$ and substituting this into Equation (4) yields

$$
\mu_{\lambda}=\frac{3 \rho_{\mathrm{d}} \int f(r) r^{2} Q_{\text {ext }}(r, \lambda) \mathrm{d} r}{4 \rho_{\mathrm{p}} \int f(r) r^{3} \mathrm{~d} r} .
$$

Including the gamma distribution (Equation (1)) to describe the frequency of particle radii, and integrating where possible over the particle radius, results in the expression

$$
\mu_{\lambda}=\frac{3 \rho_{\mathrm{d}} \int r^{\alpha+1} \mathrm{e}^{(-r / \beta)} Q_{\mathrm{ext}}(r, \lambda) \mathrm{d} r}{4 \rho_{\mathrm{p}}(\alpha+2) ! \beta^{(\alpha+3)}} .
$$

Thus, the extinction coefficient is a function of $\rho_{\mathrm{d}}, \alpha, \beta$, and $\lambda$.

Equations (6) aiil (7) assume that the radius of a sphere of cross-sec onal area equal to that of a blowing-snow particle is equal to the radius of an ice sphere of mass equal to that of the particle. This approximation presumes blowing-snow particles possessing spherical shapes and densities very near that of ice. Such an approximation has proven adequate for falling-snow optical modelling (Seagraves, 1984). Until further data become available on shape factors and densities of blowing-snow particles, correction factors cannot be confidently specified for Equations (6) and (7).

As the extinction efficiency varies within the range of blowing-snow particle sizes, precise calculation of the extinction coefficient involves defining the particle-size distribution parameters, evaluation of the Mie $Q_{\text {ext }}$, and solving for various wavelengths and drift densities. The work of Budd (1966) and Schmidt (1982) suggests that a gamma distribution with an $\alpha$ ranging from 5 to 15 is a suitable mathematical description of the suspended blowingsnow particle-radius distribution. With $\alpha$ fixed, the mean particle radius can be used to find the $\beta$ parameter (see Equation (2)). Thus, the gamma distribution can be specified by $\bar{r}$ and $\alpha$. The blowing-snow extinction coefficient for $\alpha$ equal to 5,10 , and 15 , mean particle radii equal to 40 and $90 \mu \mathrm{m}$, and a variety of wavelengths has been calculated as a function of the drift density and is shown in Figure 4. The size distributions of snow particles correspond to conditions commonly found in the suspended transport layer of blowing snow below a height of $3 \mathrm{~m}$. Variation in wavelength from ultra-violet $(300 \mathrm{~nm})$ to infra-red $(2 \mu \mathrm{m})$ results in an extinction-coefficient variation of $2.6 \%$. This variation is so small that on the scale of Figure 4 it is impossible to distinguish among the lines representing different wavelengths for a particle-radius distribution. The variation in the extinction coefficient associated with various $\alpha$ is approximately $19 \%$. This variation should be taken into account in any measurement application. A reduction in the mean particle radius from 90 to $40 \mu \mathrm{m}$ results in an approximate doubling of the extinction coefficient for a constant drift density. The mean particle size (or beta parameter of the gamma distribution of particle radii) is the most important factor affecting the blowing-snow drift density-extinction coefficient relationship for the wavelengths examined. However, in interpreting measurements made using a transmissometer, values for both the alpha and beta parameters of the gamma distribution should be addressed.

\section{VISUAL RANGE IN BLOWING SNOW}

Calculation of visibility in blowing snow is an application of the extinction of broad-band radiation by ensembles of particles. Blowing snow limits visibility because snow particles scatter and absorb electromagnetic radiation. Viewed through blowing snow, the observed contrast between sources of differing luminance is reduced (scattering) along with a reduction in illuminance (absorption). The reduction of contrast limits the distance from which objects can be distinguished.

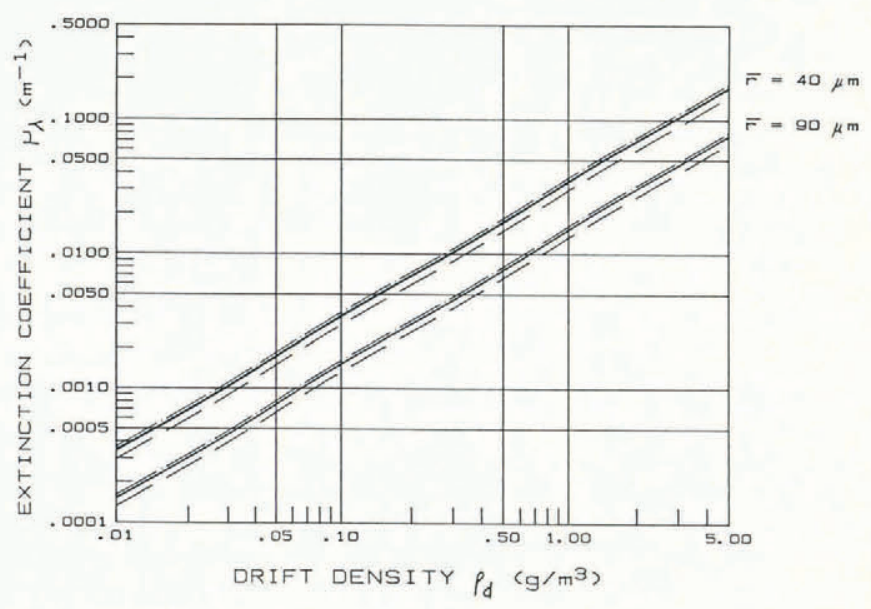

Fig. 4. Radiation extinction coefficients in blowing snow. Extinction is calculated using Mie theory for a gamma distribution of particle radii $\alpha$ of --10 and - - . 15; $\bar{r}$ of 40 and $90 \mu \mathrm{m}$; and wavelengths from $300 \mathrm{~nm}$ to $2.0 \mu \mathrm{m}$. Data for the range of wavelengths are not readily distinguished from one another at this scale. 
The meteorological visual range $V$ is a frequently and extensively measured variable; as such it is a useful index for other blowing-snow properties. $V$ is the maximum distance at which the apparent contrast between a black object and its white background is $2 \%$ of its inherent contrast. For meteorological measurements, this is considered to be the distance which a person with good vision can distinguish a black object occupying a $1^{\circ}$ solid angle with his eye. The visual range can be defined in terms of the extinction of light using Koschmieder's relationship, an inverse linear function of the extinction coefficient (Middleton, 1952) where:

$$
V=3.912 / \mu_{\text {vis }}
$$

in which $V$ is in metres when $\mu_{\text {vis }}$, the extinction coefficient for visible light (mean $\lambda=600 \mathrm{~nm}$ ), has units of $1 / \mathrm{m}$. The extinction coefficient for a fixed quantity of blowing snow does not vary substantially over the optical wavelengths. It is therefore possible to develop Equations (7) and $(8)$ into an operationally viable relationship between drift density and the meteorological visual range. It should be noted that there are large potential errors in specifying the meteorological visual range using Koschmieder's definition. The contrast threshold of $2 \%$ varies with the level of illumination and thresholds vary among observers. However, the meteorological visual range remains the standard atmospheric visibility measurement in Canada and other countries.

The mean visual extinction efficiency $\overline{Q_{\text {ext }}}$ is calculated by integrating the extinction efficiency at a wavelength of $600 \mathrm{~nm}$ over various particle-size distributions. Table III shows the mean visual extinction efficiency calculated using the Mie approximation for a particle-radius distribution $\alpha$

TABLE III. MEAN PARTICLE RADII AND CORRESPONDING MEAN VISUAL EXTINCTION EFFICIENCIES. MEAN EXTINCTION EFFICIENCIES $\overline{Q_{\text {ext }}}$ ARE CALCULATED BY INTEGRATING THE EXTINCTION EFFICIENCY AT A WAVELENGTH OF $600 \mathrm{~nm}$ OVER A GAMMA DISTRIBUTION OF PARTICLE RADII WITH $\alpha=10$. MEAN PARTICLE RADII $\bar{r}$ ARE CALCULATED FROM THE $\beta$ PARAMETER OF THE GAMMA DISTRIBUTION USING EQUATION (2) AND ASSUMING $\alpha=10$

$\begin{array}{cc}\begin{array}{c}\bar{r} \\ \mu \mathrm{m}\end{array} & \overline{Q_{\text {ext }}} \\ 30 & \\ 40 & 2.0458 \\ 50 & 2.0377 \\ 60 & 2.0324 \\ 70 & 2.0287 \\ 80 & 2.0259 \\ 90 & 2.0237 \\ & 2.0219\end{array}$

parameter of 10 and a range of mean particle radii (note $\bar{r}=\alpha \times \beta)$. The striking aspect of these results is the limited variation in the extinction efficiency for typical non-uniform distributions of blowing-snow particle sizes. For a specific particle-size distribution $\overline{Q_{\text {ext }}}$ is a constant and can be calculated as a function of the mean particle radius if the shape parameter of the radius distribution is estimated. A regression analysis on the results in Table III yields a simple, approximate relationship between the mean particle radius $\bar{r}$ and the mean extinction efficiency $\overline{Q_{\text {ext }}}$ at a wavelength of $600 \mathrm{~nm}$ and a radius distribution $\alpha$ of 10 , where

$$
\overline{Q_{\text {ext }}}=1.82 \bar{r}^{-0.011}
$$

and the coefficient of determination is 0.99 .

Using the results of Equation (9) or the geometrical optics approximation of 2.0 , Equation (7) can now be fully integrated, combined with Equation (8), and expressed in terms of the visual range, drift density, and mean particle radius, where

$$
V=\frac{5.217 \bar{r} \rho_{\mathrm{p}}}{\rho_{\mathrm{d}} \overline{Q_{\mathrm{ext}}} c_{\alpha}}
$$

and

$$
c_{\alpha}=\frac{(\alpha+1) ! \alpha}{(\alpha+2) !} .
$$

The term $c_{\alpha}$ is the gamma-distribution shape-correction factor. Values of $c_{\alpha}$ vary from 0.7413 for $\alpha=5$ to 0.8333 for $\alpha=10$ and 0.8824 for $\alpha=15$. For $\alpha=10$, the per cent difference between the exact solution for the visual range as calculated using Equations (7) and (8) and that calculated using Equation (10) is less than $0.3 \%$, the difference due to the approximation using Equation (9) which assumes $\alpha=10$ when calculating $\overline{Q_{\text {ext }}}$. Use of the geometrical optics approximation of $2.0^{\circ}$ for the extinction efficiency results in an added error which varies from $1 \%$ for a $\bar{r}$ of $90 \mu \mathrm{m}$ to $2 \%$ for a $\bar{r}$ of $30 \mu \mathrm{m}$.

The meteorological visual range in blowing snow has been calculated using Equation (10) and for precision, Equation (9), for $\alpha=10$ and a variety of mean particle radii and drift densities. The results are shown in Figure 5. The mean particle size has a significant effect on the visual range-drift density relationship. Meteorological visual range

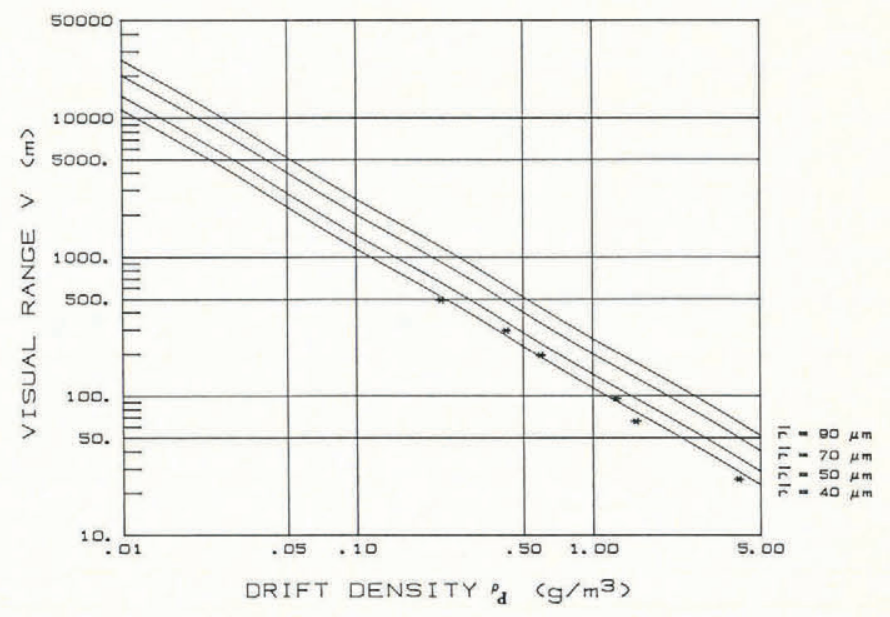

Fig. 5. Visual range in blowing snow. Extinction is calculated using an $\alpha$ of 10 and $\bar{r}$ of 40, 50, 70, and $90 \mu \mathrm{m}$. Budd and others' (1966) observations are plotted as (*).

following Middleton's (1952) specification and the drift density at $2 \mathrm{~m}$ height were precisely measured in the Antarctic during blowing snow by Budd and others (1966). Their measurements are also plotted in Figure 5 and correspond to the theoretical predictions for a mean particle radius of about $40 \mu \mathrm{m}$. Mellor (1965) noted that the mean particle radius of blowing-snow particles at visual height $(2 \mathrm{~m})$ during the experiments of Budd and others is $44 \mu \mathrm{m}$.

\section{DISCUSSION}

Appreciable variation in the extinction efficiency of monochromatic radiation is manifest by individual blowingsnow particles of small size. For example, the extinction efficiency for blowing-snow particles in near-infra-red radiation varies irregularly over a range of about $5 \%$ for particles of less than $40 \mu \mathrm{m}$ and about $10 \%$ for particles of less than $15 \mu \mathrm{m}$ radius (see Fig. 2d). Shorter wavelengths exhibit an irregular variation in the extinction efficiency of a lesser magnitude. This effect must severely limit the accuracy of size estimates made from the transmittance of monochromatic infra-red radiation. For the full range of blowing-snow particle sizes (down to 20 or $30 \mu \mathrm{m}$ radius), the amplitude of the extinction efficiency for monochromatic radiation limits the accurate interpretation of 
extinction measurements to those using wavelengths of less than $1.06 \mu \mathrm{m}$. Monochromatic radiation measurements interpreted using geometrical optics should be limited to wavelengths less than $600 \mathrm{~nm}$, though particle sizes typical of saltating snow permit use of the full visible band.

For radiation possessing a range of wavelengths, errors of an appreciable magnitude can occur when approximating the extinction efficiency of a single blowing-snow particle by geometrical optics. However, the errors are less than those for monochromatic radiation. These effects are most pronounced for small blowing-snow particles and long wavelengths. In an example using near-infra-red radiation (mean $\lambda=1.06 \mu \mathrm{m}$ ), the smallest snow-particle radius for which errors in applying geometrical optics are less than $5 \%$ is $32 \mu \mathrm{m}$ for monochromatic radiation; for broad-band radiation the corresponding particle radius is $16 \mu \mathrm{m}$. A particle detector using visible light calibrated with geometrical optics experiences errors of less than $5 \%$ for particle radii down to $9 \mu \mathrm{m}$. However, the limiting radius for errors of less than $2 \%$ is $31 \mu \mathrm{m}$. As a typical mean blowing-snow particle radius at $2 \mathrm{~m}$ height is $40 \mu \mathrm{m}$, it is a matter of judgement based on the detector application whether use of the Mie theory calibration is justified. Obviously, for many particle sizes and wavelengths the geometrical optics approximation is sufficiently accurate for measurement applications. However, designers and operators of particle detectors using broad-band radiation should consider using Mie extinction values to calibrate the instruments for particle sizes and wavelengths where Table II indicates unacceptable errors in the geometrical optics approximation.

For the wavelengths examined, there is a very limited variation in the extinction efficiency of ensembles of blowing-snow particles possessing typical size distributions. A change in the wavelength from ultra-violet to near-infrared changes the extinction coefficient by $2.6 \%$. This magnitude of change can be neglected in most measurement and prediction applications. For a gamma distribution fit to a non-uniform distribution of blowing-snow particle radii, the extinction coefficient varies $19 \%$ as the shape parameter $\alpha$ varies from 5 to 15 . This variation is notable and worth accounting for in the interpretation of output from transmissometers. A reduction in the mean particle radius from 90 to $40 \mu \mathrm{m}$ results in an approximate doubling of the extinction coefficient for a constant drift density. The mean particle size (or beta parameter in terms of the gamma distribution) is the most important factor affecting the blowing-snow drift density-extinction coefficient relationship for the wavelengths examined.

For blowing snow at "eye-level" height in ambient light, there is only a $0.3 \%$ difference between the distributed and mean Mie extinction calculation, and only a 1 or $2 \%$ difference between the mean Mie calculation and the geometrical optics approximation. Use of the geometrical optics approximation in visual range calculations should exhibit sufficient accuracy for most applications. However, the alpha parameter of the gamma distribution and mean particle radius are important in visual range modelling and should be estimated where visual range is predicted from drift density of particle-number density measurements. Budd and others' (1966) measurements of visual range can be successfully modelled when particle size is accounted for.

\section{CONCLUSIONS}

For calculations of extinction where detection of scattered radiation is not considered, the approximation of blowing-snow particles as ice spheres results in acceptably small errors for most applications.

For monochromatic radiation possessing wavelengths longer than the ultra-violet, geometrical optics approximations lead to errors of greater than $4 \%$ in calculations of extinction by single particles of sizes comprising a significant component to suspended blowing snow. Prediction of extinction for small particles becomes difficult even with Mie theory in cases where the extinction efficiency varies more than $5 \%$ from 2.0. This limits the usefulness of near-infra-red radiation in blowing-snow particle detection.

For broad-band radiation, the geometrical optics approximation is sufficiently accurate for many singleparticle measurement instruments and applications. Designers and operators of particle detectors using broad-band radiation should consider using $\mathrm{Mie}$ calculations to calibrate the instruments for wavelengths in the infra-red band.

For typical blowing-snow particle-size distributions, use of the geometrical optics approximations in broad-band extinction and visual range calculations exhibits sufficient accuracy for most applications. The alpha (shape) parameter and the beta parameter (scale parameter or its surrogate, the mean particle radius) of the gamma-distribution of particle radii are very important in broad-band extinction and visual range modelling. Applications which estimate blowing-snow quantities from broad-band extinction measurements or estimate visual range from blowing-snow quantities should address the shape and mean value of the snow-particle radius distribution.

\section{ACKNOWLEDGEMENTS}

The authors wish to thank Dr D.M. Gray, Division of Hydrology, University of Saskatchewan, Saskatoon; Dr J. Dozier, University of California, Santa Barbara; Dr R.A Schmidt, Rocky Mountain Forest and Range Experiment Station, Fort Collins, Colorado, and anonymous referees for helpful discussions regarding this paper, and E. Wigham, Division of Hydrology, for preparation of the manuscript The research was supported by the Natural Sciences and Engineering Research Council of Canada, operating grant No. A4363, and the first author was assisted by Saskatchewan Research Council Graduate Studies Scholarships.

\section{REFERENCES}

Budd, W.F. 1966. The drifting of nonuniform snow particles. In Rubin, M.J., ed. Studies in Antarctic meteorology. Washington, DC, American Geophysical Union, 59-70. (Antarctic Research Series 9.)

Budd, W.F., Dingle W.R.J., and Radok, U. 1966. The Byrd Snow Drift Project: outline and basic results. In Rubin, M.J., ed. Studies in Antarctic meteorology. Washington, DC, American Geophysical Union, 71-134. (Antarctic Research Series 9.)

Gubler, H. 1981. An electronic remote snow-drift gauge. Journal of Glaciology, 27(95), 164-74

Haan, C.T. 1977. Statistical methods in hydrology. Ames, IA, The Iowa University Press.

Hodkinson, J.R. 1963. Light scattering and extinction by irregular particles larger than the wavelength. In Kerker, M., ed. Electromagnetic scattering. New York, Macmillan, 89-100.

Hulst, H.C. van de. 1957. Light scattering by small particles. New York, John Wiley and Sons.

Landon Smith, I.H., and Woodberry, B. 1965. The photoelectric metering of wind-blown snow. ANARE Interim Reports, Ser. A(IV), Glaciology, 1-18. (Publication 79.)

Mellor, M. 1965. Blowing snow. CRREL Monograph III A3c.

Mellor, M. 1966. Light scattering and particle aggregation in snow-storms. Journal of Glaciology, 6(44), 237-48.

Middleton, W.E.K. 1952. Vision through the atmosphere. Toronto, University of Toronto Press.

Mie, G. 1908. Beiträge zur Optik trüber Medien, speziell kolloidalen Metall-Lösungen. Annalen der Physik, 25, 377-445.

Nussenzveig, H.M., and Wiscombe, W.J. 1980. Efficiency factors in Mie scattering. Physical Review Letters, 45(18), 1490-94.

Pollak, J.B., and Cuzzi, J.N. 1980. Scattering by nonspherical particles of size comparable to a wavelength: a new semi-empirical theory and its application to tropospheric aerosols. Journal of the Atmospheric Sciences, 37, 868-81.

Pomeroy, J.W., and Male, D.H. 1985. Optical properties and detection of blowing snow. EOS. Transactions of the American Geophysical Union, 66(46), 902.

Pomeroy, J.W., and Male, D.H. 1987. Wind transport of seasonal snowcovers. In Seasonal snowcovers: physics, chemistry, hydrology. Dordrecht, D. Reidel Publishing Co. (NATO Advanced Study Institute Ser. C211.) 
Schmidt, R.A. 1977. A system that measures blowing snow. Fort Collins, CO, U.S. Department of Agriculture. Forest Service. (Research Paper RM-194.)

Schmidt, R.A. 1979. Measuring visibility in blowing snow. In Snow Removal and Ice Control Research. Proceedings of the Second International Symposium, held May 15-19. 1978, at Hanover, New Hampshire. Washington, DC, National Academy of Sciences. Transportation Research Board, 200-07. (Special Report 185.)

Schmidt, R.A. 1981. Estimates of threshold windspeed from particle sizes in blowing snow. Cold Regions Science and Technology, 4(3), 187-93.

Schmidt, R.A. 1982. Vertical profiles of windspeed, snow concentration, and humidity in blowing snow. Boundary-Layer Meteorology, 23(2), 223-46.

Schmidt, R.A. 1984. Measuring particle size and snowfall intensity in drifting snow. Cold Regions Science and Technology, 9(2), 121-29.

Seagraves, M.A. 1981. Some optical properties of blowing snow. White Sands Missile Range, NM, U.S. Army Electronics Research and Development Command. (U.S. Army Atmospheric Sciences Laboratory Report ASL-TR-0091.)

Seagraves, M.A. 1984. Precipitation rate and extinction in falling snow. Journal of the Atmospheric Sciences, 41(11), 1827-35.

Shiotani, M., and Arai, H. 1952. A short note on the snow-storm. In Proceedings of the Second Japan National Congress for Applied Mechanics. Tokyo, Science Council of Japan, 217-18.

Tabler, R.D. 1979. Visibility in blowing snow and applications in traffic operations. In Snow Removal and Ice Control Research. Proceedings of the Second International Symposium, held May 15-19, 1978, at Hanover, New Hampshire. Washington, DC, National Academy of Sciences. Transportation Research Board, 208-14. (Special Report 185.)

Tabler, R.D. 1984. Using visual range data for highway operations in blowing snow. Optical Engineering, 23(1), 55-61.

Ungut, A., Grehan, G., and Goesbet, G. 1981. Comparisons between geometrical optics and Lorenz-Mie theory. Applied Optics, 20(17), 2911-18.

Warren, S.G. 1984. Optical constants of ice from the ultraviolet to the microwave. Applied Optics, 23(8), 1206-25.

Wiscombe, W.J. 1980. Improved Mie scattering algorithms. Applied Optics, 19(9), 1505-09. 